\title{
Skills training in bariatric surgery
}

\section{Dimitrios Stefanidis M.D., Ph.D., F.A.C.S., F.A.S.M.B.S.}

Department of Surgery, School of Medicine Indiana University, Indianapolis, Indiana

Zevin et al. created a comprehensive skills curriculum for bariatric surgery to address cognitive, technical, and nontechnical skills and assessed the curriculum’s effectiveness in their residency program [1]. Using a single-blinded, randomized controlled design, they allocated 20 intermediate-level residents either to an intervention group that trained to proficiency using the new curriculum or to a control group. They assessed participant knowledge, technical skills, and nontechnical skills in a live, anesthetized porcine model and in the human operating room. They found significant improvements in technical and nontechnical skills of the intervention group compared with the control group during assessment on the pig model; they found similar technical skills and superior nontechnical skills in the intervention group compared with those of chief residents in the same program [1].

The authors need to be congratulated not only for developing a comprehensive skills curriculum for bariatric surgery but also for providing level I evidence of its effectiveness. The comprehensive nature of their curriculum stands out compared with similar curricula in the literature. In addition to incorporating cognitive knowledge and simulator training in technical skills, which is today’s standard in most available skills curricula, they also addressed nontechnical skills. The importance of including nontechnical skills such as situation awareness, decision-making, communication and teamwork, and leadership in their curriculum is evidenced by studies that have found that these skills not only directly affect technical skills [2] but also influence patient safety [3]. Although it is not possible to glean from this study what the actual effect of nontechnical skills training was on resident overall performance, given its importance, nontechnical skill teaching should be part of all skills curricula.

This is the author's manuscript of the article published in final edited form as:

Stefanidis, D. (2017). Skills training in bariatric surgery. Surgery for Obesity and Related Diseases, 13(5), 824-825. https://doi.org/10.1016/j.soard.2017.01.036 
Additional evidence of the quality of this study is use of a proficiency-based skills curriculum for technical skill training. Proficiency-based training or mastery learning is the current gold standard in simulator training. This type of training sets expert-derived training goals and provides timely performance feedback to the learner [4,5], both important factors that promote deliberate practice, which is necessary for the acquisition of expertise [6]. Perhaps one of the biggest advantages of this type of training is that it is tailored to the unique needs of each learner. Instead of defining a number of repetitions or procedures that need to be done as traditional training mandates, it starts with the end goal in mind: The learner needs to practice until the required level of performance has been achieved, independent of how long or how many practice sessions it takes [4]. Although this type of training has become the standard in surgical skills curricula, its implementation into the main residency curriculum has yet to be accomplished in the United States. Studies such as this and others clearly provide the evidence we need to come closer to adopting proficiency based training for our resident curriculum [5]. It may be the solution to the commonly voiced concern of significant skill variability and lack of competency of graduating surgery residents [7].

The equivalency of demonstrated technical skill between intervention group residents and chief residents may have additional implications for resident curriculum design. If using simulation and effective skills curricula such as this presented by Zevin et al. enables residents to achieve a level of performance or proficiency in a procedure sooner than traditional training allows, then this could be the basis of training acceleration. Residents who follow such training paradigms may not only be able to compensate for the decreased training opportunities that work hour limitations have imposed on them but even complete training sooner than traditional training has ever allowed. Such a training paradigm might make possible compressing general surgery training into fewer than 5 years and allow for fellowships to start sooner, as has been suggested previously [8].

The curriculum presented did not lead to any improvement in knowledge of the intervention group, but this appears to have been because only a small proportion of participants actually completed the online 
cognitive modules. It would have been advisable to mandate completion of the cognitive component before starting technical and nontechnical skills training. It should also be noted that the procedure this curriculum provided effective training for was laparoscopic jejunojejunostomy and not the whole laparoscopic gastric bypass procedure. Nevertheless, I have no doubt that enriching the existing curriculum with additional procedural steps of the lap gastric bypass would have led to a very similar effect on resident training.

In summary, the paper by Zevin et al. provides our field with a novel, comprehensive curriculum for training in aspects of laparoscopic gastric bypass and evidence of its effectiveness. It can serve as a template for future development of similar skills curricula in bariatric surgery and surgery in general. 


\section{References}

[1] Zevin B, Dedy NJ, Bonrath EM, Grantcharov TP. Comprehensive simulation-enhanced training curriculum in bariatric surgery: a randomized controlled trial. Surg Obes Relat Dis 2016. In press.

[2] Hull L, Arora S, Aggarwal R, Darzi A, Vincent C, Sevdalis N. The impact of nontechnical skills on technical performance in surgery: a systematic review. J Am Coll Surg 2012;214(2):214-30.

[3] Riley W, Davis S, Miller K, Hansen H, Sainfort F, Sweet R. Didactic and simulation nontechnical skills team training to improve perinatal patient outcomes in a community hospital. Jt Comm J Qual Patient Saf 2011;37(8):357-64.

[4] Stefanidis D. Optimal acquisition and assessment of proficiency on simulators in surgery. Surg Clin North Am 2010;90(3):475-89.

[5] McGaghie WC. Mastery learning: it is time for medical education to join the 21st century. Acad Med 2015;90(11):1438-41.

[6] Ericsson KA. Acquisition and maintenance of medical expertise: a perspective from the expertperformance approach with deliberate practice. Acad Med 2015;90(11):1471-86.

[7] Mattar SG, Alseidi AA, Jones DB, et al. General surgery residency inadequately prepares trainees for fellowship: results of a survey of fellowship program directors. Ann Surg 2013;258(3):440-9.

[8] Debas HT, Bass BL, Brennan MF, et al. American Surgical Association Blue Ribbon Committee Report on Surgical Education: 2004. Ann Surg 2005;241(1):1-8. 